\title{
ON THE DIMENSION OF THE SOLAR ACTIVITY ATTRACTOR
}

\author{
C.M. ORZARU \\ Astronomical Institute of the Romanian Academy \\ Str. Cutitul de Argint 5, 75212 Bucuresti 28 \\ Romania
}

\begin{abstract}
The correlation dimension $D^{(2)}$ as a characteristic measure of the regular or chaotic behaviour of the solar dynamical system has been calculated. The algorithm suggested by Grassberger and Procaccia (1983) has been applied to time series of relative sunspot numbers and of areas of sunspots and faculae. In the first case, a correlation dimension $D^{(2)} \simeq 1.5$ has been found; in the other two cases, the algorithm was not convergent, the results obtained being not relevant, due to the too short series of data available.
\end{abstract}

\section{Introduction}

The question whether the solar cycle exhibits or not a stochastic behaviour, did not receive yet a definitive answer.

A good "measure" of the global solar activity are the sunspot relative numbers, indicator introduced by $\mathrm{R}$. Wolf.

Although known as the "11-year cycle", the sunspot cycle presents long-term variations both in amplitude and period, which cannot be predicted at present.

The cyclicity of solar activity is well explained by the linear dynamo theory, but the appearance of irregular minima (as those of Maunder, Sporer and the Mediaeval one) requires a nonlinear model. Zeldovich and Ruzmaikin (1983) suggested a nonlinear system of ordinary differential equations which have strange attractor solutions; these ones exist for dissipative dynamical systems and characterize a chaotic (but deterministic) evolution in the phase space.

A quantitive characterization is given by the attractor's Hausdorff dimension, Lyapunov exponents and Kolmogorov-Sinai entropy. In this paper, a correlation dimension study will be applied to time series of relative sunspot numbers and of sunspots and faculae areas. The proportionality between the total magnetic flux in a sunspot and its area enables the use of the latter one as a crude estimate of the total magnetic flux at the surface of the Sun. Faculae are magnetic features characterizing convection's intensity in the layer below the photosphere.

Other estimates of the attractor dimensions were made by Kurths (1987 a,b and 1991) for sunspot numbers and for solar radio pulsations which often occur during solar flares.

\section{Estimation of the correlation dimension}

In the following, the Grassberger-Procaccia algorithm (1983) will be used to determine the correlation dimension by calculating the exponent of the spatial correlation function for three sets of experimental data.

For a dissipative dynamical system evolving deterministically, its trajectory lies on a submanifold of the total phase space. 
The Whitney theorem (1936) assesses that d-dimensional differentiable manifolds of the $n$-dimensional phase space, can be embedded into an $(2 d+1)$-dimensional space.

The theorem, valid for $d$ integer, has not yet been proved for the casc of fractal sets (which are not manifolds).

The technique of estimating the correlation dimension will be described in the following.

A time series of a measured physical variable $\left(x_{i}\right)_{1 \leq i \leq N}(N$ being the number of data) is used to reconstruct the attractor.

This means a vector:

$$
x_{i}=\left(x\left(t_{i}\right), x\left(t_{i}+m\right), \ldots, x\left(t_{i}+(d-1) m\right)\right.
$$

where $i=1,2, \ldots, N-d+1, d$ is the embedding dimension and $m$ the time delay.

The attractor thus reconstructed from a single time variable is a d-dimensional projection of the original one. The number of points inside a ball of radius $r$ centred at a reference point $x_{j}$ is counted and the q-th order correlation integral calculated:

$$
C_{d}^{(q)}(r)=\left\{(1 / N) \sum_{i=1}^{N}\left[(1 / N) \sum_{j=1}^{N} H\left(r-\left|x_{i}-x_{j}\right|\right)\right]^{q-1}\right\}^{1 /(1-q)}
$$

( $H(x)$ is the Heaviside function, $H(x)=0$ for $x \leq 0$ and $H(x)=1$ for $x>0$ ). The slope of the correlation integral defines the q-th order dimension $D^{(q)}$ :

$$
D^{(q)}=\lim _{r \rightarrow 0} \frac{\dot{\ln C} C_{d}^{(q)}(r)}{\ln r}
$$

Next step is incrementing $d$ and calculating $C_{d=2}^{q}(r)$ and so on until the slopes does not vary significantly.

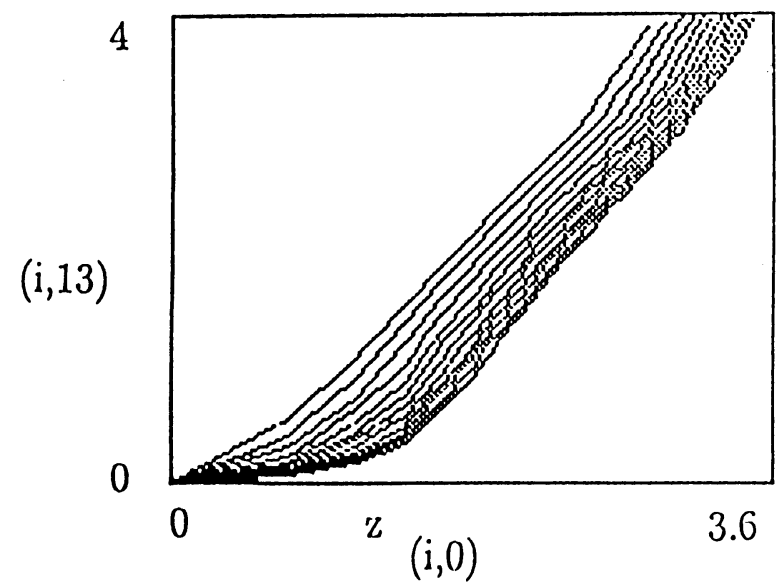

Fig.1. The typical $\ln C$-lnr dependence for the relative sunspot numbers 
Then a graph of $\ln C(r)$ is plotted against $\ln r$, trying to identify a "scaling region", i.e. a straight line segment over a reasonable range of $\ln r$. For $q=2$, we obtain the so-called "correlation dimension" $D^{(2)}$. This quantity characterizes the distribution of the points on the set. The mathematical constraints arc: a long series of data is necessary to ensure the convergence of the algorithm $\left(D^{(2)}\right.$ being an asymptotic measure), the necessity that points lic on the attractor (not being transient) and a good coverage of the whole attractor (Judd, 1991 and Eckmann and Ruelle, 1985).

I used the Grassberger-Procaccia method for sunspot numbers recorded between 1749-1991 (2904 data) and monthly averaged areas of sunspots and faculac, recorded between 1874-1954. The results are shown in Fig. 1-3. Similar results were obtained by Kurths (1987a) for sunspot numbers (see also Ruzmaikin 1983, 1990).

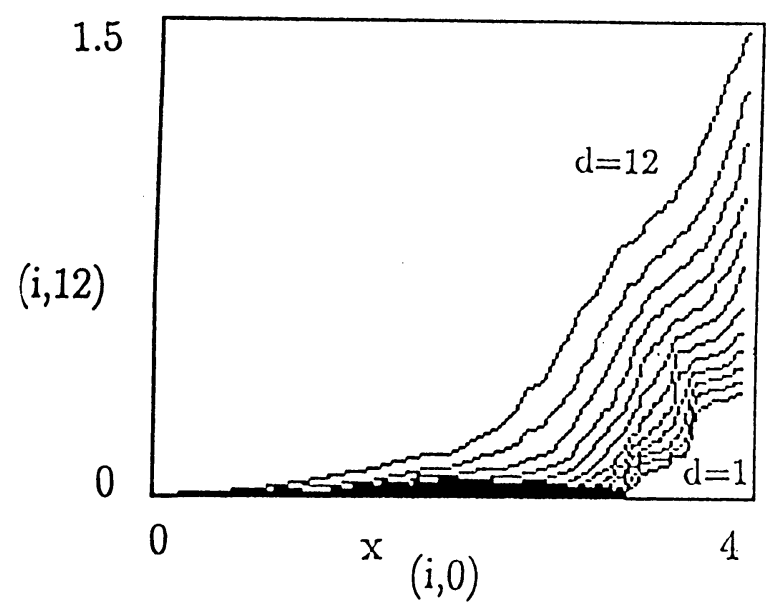

Fig. 2. The dependence $\ln C$-lnr for sunspot areas

\section{Discussions}

For the sunspot numbers the correlation dimension of about 1.5 was obtained for $d=2, \ldots, 12$ after eliminating the regions affected by noise.

I used as indicators sunspot and faculac areas because their magnetic flux gives a good characterization of the global solar activity; unfortunatcly, in these cases the algorithm was not convergent, due to the too short serics of data.

The graphic from Fig. 1 shows the dependence of the correlation dimension on the choice of the scaling region and the increasing degree of corrclation with increasing $r$.

The slopes seem to converge with increasing d but the time scries is still too short to be sure that the value of $D^{(2)}$ obtained is an accurate one. For the same reason, we are not sure that a good coverage of the whole attractor is ensured.

A low-dimensional attractor seems to exist in the phase space, but it must be emphasized that the existence of a chaotic attractor in the phase space of the 
Then a graph of $\ln C(r)$ is plotted against $\ln r$, trying to identify a "scaling region", i.c. a straight linc scgmenl over a reasonable range of $\ln r$. For $q=2$, we obtain the so-called "correlation dimension" $D^{(2)}$. This quantity charactcrizes the distribution of the points on the set. The mathematical constraints arc: a long series of data is necessary to cnsurc the convergence of the algorithm $\left(D^{(2)}\right.$ being an asymptotic measurc), the necessity that points lic on the attractor (not bcing transient) and a good coverage of the wholc attractor (Judd, 1991 and Eckmamn and Ruelle, 1985).

I used the Grassberger-Procaccia method for sunspot numbers recorded betwecn 1749-1991 (2904 data) and monthly averaged arcas of sunspots and faculac, recorded between 1874-1954. The results arc shown in Fig. 1-3. Similar results wcre obtained by Kurths (1987a) for sunspot numbers (scc also Ruzmaikin 1983, 1990).

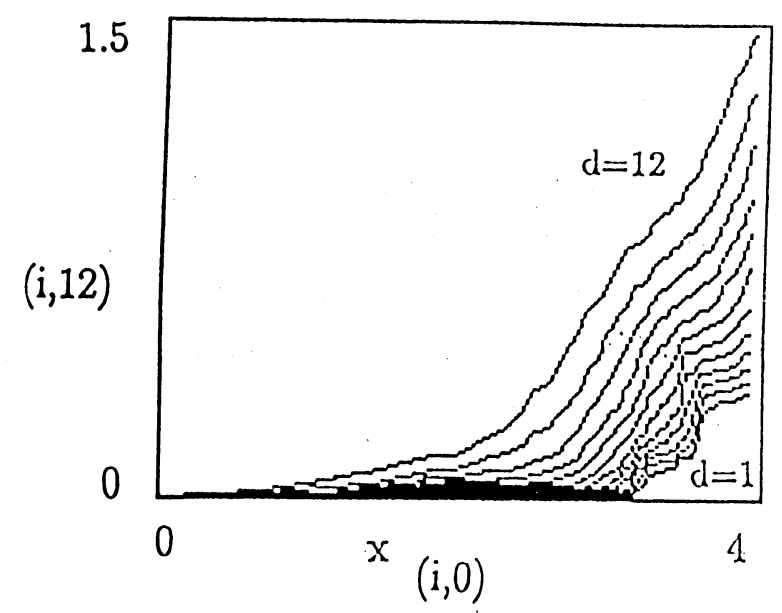

Fig. 2. The dependence ln $C$-lnr for sunspot arcas

\section{Discussions}

For the sunspot numbers the corrclation dimension of about 1.5 was obtained for $d=2, \ldots, 12$ after eliminating the regions affected by noisc.

I used as indicators sunspot and faculac arcas bccausc their magnetic flux gives a good characterization of the global solar activity; unfortunatcly, in these cascs the algorithm was not convergent, duc to the too short serics of data.

The graphic from Fig. 1 shows the dependence of the corrclation dimension on the choice of the scaling region and the increasing degrec of corrclation with increasing $r$.

The slopes seem to converge with increasing $d$ but the lime scrics is still too short to be sure that the value of $D^{(2)}$ obtained is an accurate one. For the same reason, we are not sure that a good coverage of the wholc attractor is ensured.

A low-dimensional attractor secms to exist in the phase space, but it must be emphasized that the existence of a chaotic altractor in the phase space of the 
dynamical system has not yet a rigorous mathematical proof.

A forthcoming paper will be completed with a calculus of maximum Lyapunov exponent and of the Kolmogorov entropy.

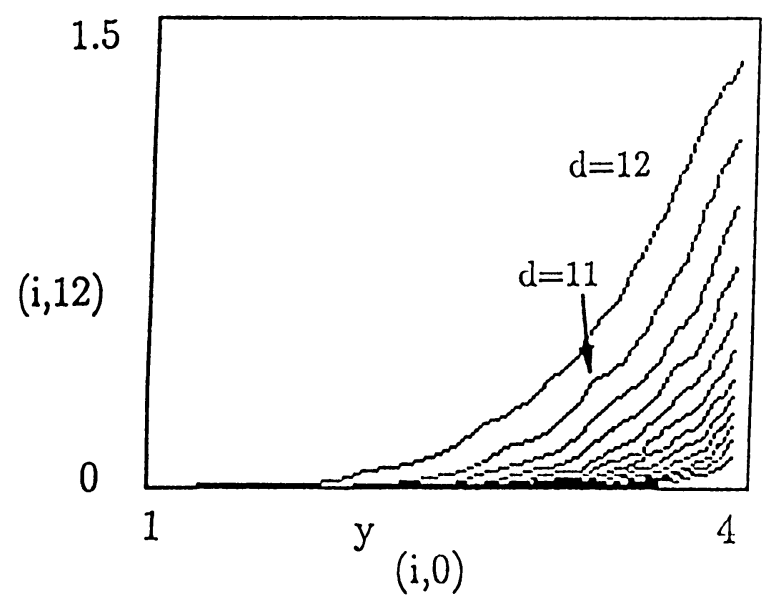

Fig. 3. The dependence $\ln C$ - In for faculae areas

\section{Acknowledgements}

I thank Dr. Jürgen Kurths for the suggestions and valuable discussions.

\section{References}

Eckmann, J.P., Ruelle, D.: 1985, Rev. Mod. Phys. 57, 617.

Grassberger, P., Procaccia, I., 1983, Phys. Rev. Lett. 50, 346.

Judd, K., Mees, A.I., 1991, Intern. Journal of Bifurcation and Chaos, Vol. 1, No. 2, 467-470.

Kurths, J., 1987, preprint ZIAP

Kurths, J., Herzel, H., 1987, Physica 25D, 165-172.

Kurths, J., Benz, A.O., Aschwanden, M.J., 1991, A stron. Astrophys. 248, 270-276.

Ruzmaikin, A.A., 1990, in IAU Symp. 138 Solar Photosphcre:Structure, Convection and Magnetic Fields, J.O. Stenflo ed., 343-353.

Zeldovich, Y.B., Ruzmaikin, A.A., 1983, Astrophys. and Spacc Phys. Rcv., Vol. 2, 333-383. 